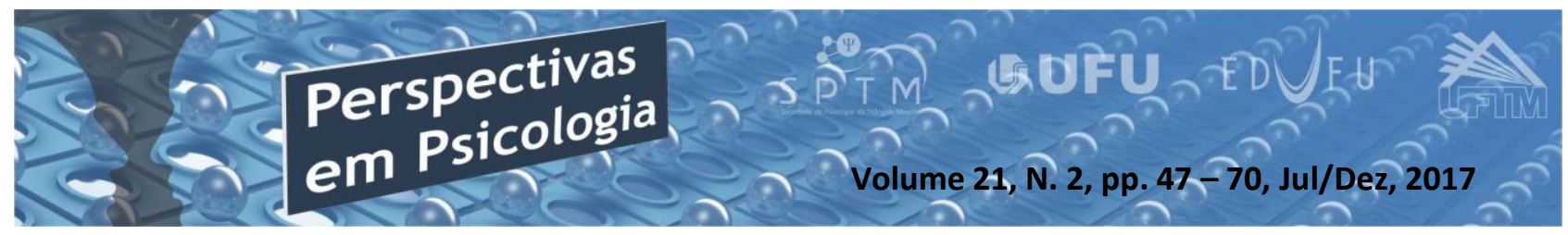

\title{
FADIGA SECUNDÁRIA À QUIMIOTERAPIA EM MULHERES COM CÂNCER DE MAMA: REVISÃO INTEGRATIVA DE LITERATURA
}

\author{
Anna Cláudia Yokoyama dos Anjos \\ Cristiane Soares Campos \\ Nayara Ferreira Cunha \\ Carine Ferreira Lopes \\ Luana Luiza Alves \\ Juliana Pena Porto \\ (Universidade Federal de Uberlândia, UFU, Uberlândia - MG)
}

\begin{abstract}
Resumo
Objetivou-se identificar resultados de estudos primários que investigaram a fadiga secundária à quimioterapia, em mulheres com câncer de mama. Trata-se de revisão integrativa de literatura, com busca nas bases de dados BVS, CINAHL, PUBMED, SCIELO, PsycINFO, MEDLINE, Scopus, LILACS. Selecionou-se 12 artigos, foram identificados cinco temas: Impacto da prática de exercícios físicos sobre a fadiga secundária à quimioterapia; relação entre a fadiga e outros sintomas decorrentes da quimioterapia; alterações epigenéticas associadas à fadiga secundária à quimioterapia; utilização de medicina complementar e alternativa para tratamento da fadiga secundária à quimioterapia; trajetória da fadiga durante a quimioterapia para câncer de mama. Esta revisão possibilitou melhor compreensão acerca da temática, trazendo contribuições significativas para a construção do conhecimento e para a prática assistencial.
\end{abstract}

Palavras-chave: fadiga; neoplasias da mama; quimioterapia; revisão; Enfermagem.

\section{Abstract \\ Secondary Fatigue Due to Chemotherapy in Women with Breast Cancer: Integrative Literature Review}

This study was aiming to identify the results of primary studies based investigation on secondary fatigue due to chemotherapy in women suffering from breast cancer. This is an integrative literally revision with sources from databases such as BVS, CINAHL, PUBMED, SCIELO, PsycINFO, MEDLINE, Scopus, LILACS. Twelve articles were selected, five themes were identified: The physical exercise impact on secondary fatigue due to chemotherapy; The relationship between fatigue and other symptoms arising from chemotherapy; The epigenetic changes associated with secondary fatigue due to chemotherapy; The use of complementary and alternative medicine in order to treat secondary fatigue due to chemotherapy; The fatigue trajectory during chemotherapy for breast cancer. Thanks to this review, it was possible to grasp a better understanding of the theme in general, adding meaningful contributions to the knowledge on this issue and to help provide a better practice care.

Keywords: fatigue; breast neoplasms; drug therapy; review; nursing. 


\section{Introdução}

O câncer de mama é o tipo que possui maior incidência e mortalidade na população feminina em todo o mundo, independentemente do grau de desenvolvimento dos países. No Brasil são esperados 57.960 casos novos de câncer de mama para o biênio 2016-2017. (Brasil, 2015).

As mulheres com câncer de mama experimentam mudanças significativas em sua qualidade de vida, que se devem ao impacto causado pela própria doença e pelos efeitos adversos e colaterais primários e secundários do tratamento proposto. (Nicolussi, \& Sawada, 2011).

Dentre as opções terapêuticas para o câncer de mama, a quimioterapia (QT) atualmente apresenta-se como opção de escolha para maioria dos casos a qual, devido à inespecificidade dos fármacos antineoplásicos, causa reações que comprometem a vida dessas pacientes. (Machado, \& Sawada, 2008). Os efeitos adversos mais relevantes decorrentes da QT são: depressão, ansiedade, insônia, fadiga, estresse psicológico, alterações hematológicas, gastrointestinais e do tecido tegumentar e limitações funcionais. (Martins et al., 2009).

A fadiga relacionada ao câncer (FRC) é considerada o sintoma mais prevalente e penoso em relação ao tratamento, o qual tem mostrado alta prevalência durante a QT e pode estar presente até cinco anos após o tratamento, sem melhora durante os dois primeiros anos. (Husebo, Dyrstad, Mjaaland, Soreide, \& Bru, 2014). A FRC impacta nas atividades diárias, nas relações sociais e na qualidade de vida da mulher com câncer de mama submetida à QT; também tem sido considerada como preditor de sobrevida para estas pacientes. (Machado, \& Sawada, 2008).

A prevalência de fadiga estimada varia de forma abrangente, refletindo uma variedade de populações nas quais o sintoma tem sido estudado. Afeta de $70 \%$ a $100 \%$ dos pacientes

que recebem drogas quimioterápicas, causando assim substancial impacto funcional e psicológico; ressalta-se a condição de que é raramente discutida e tratada. (Bonassa, \& Oliveira, 2012).

A FRC é diferente da fadiga típica. Enquanto a fadiga típica é descrita como uma sensação de cansaço agudo, que sinaliza um desequilíbrio entre descanso e atividade, com melhora após a interrupção da ação fatigante ou após o repouso e que acomete indivíduos saudáveis; a FRC tem sido definida como uma condição angustiante, persistente, caracterizada por estresse, cansaço físico, emocional e/ou 
cognitivo, exaustão, diminuição da concentração, declínio da capacidade funcional, entre outros, relacionado ao câncer ou ao seu tratamento. (ABCP, 2010; Ancoli-Israel et al., 2012).

A FRC ainda pode ser classificada como primária ou secundária. A primeira é decorrente da própria doença, ou seja, faz parte do quadro clínico independente da ação de outros fatores não relacionados diretamente à doença; já a segunda é decorrente de síndromes concomitantes, comorbidades ou do próprio tratamento da doença base como, por exemplo, a quimioterapia. (ABCP, 2010).

A fadiga secundária à quimioterapia (FSQ) tem presença constante durante esta modalidade de tratamento, sendo considerada um dos efeitos adversos da QT com duração mais longa e maior intensidade de sintomas relacionados. Apresenta aumento significativo com os ciclos consecutivos de QT, sendo verificado em mais de $75 \%$ dos pacientes, relatando sensação de cansaço debilitante e perda de energia. (Carr, \& Vissers, 2014; Curt et al., 2000). Em alguns casos, a FSQ é a barreira mais significativa para recuperação funcional de pacientes que estão estáveis em relação à progressão da doença durante a QT. (Curt et al., 2000).
Deste modo, conhecer as implicações e repercussões da FSQ em mulheres submetidas à esta terapêutica, auxiliará enfermeiros e toda a equipe de saúde no planejamento de assistência qualificada, que visa a prevenção e/ou diminuição desse e de outros efeitos adversos, bem como na elaboração de estratégias que melhorem a funcionalidade e o bem-estar dessas pacientes. (Machado, \& Sawada, 2012; Meneses-Echávez, González-Jiménez, Correa-Bautista, Ramírez-Vélez, \& Valle, 2015).

A assistência pautada em conclusões, geradas a partir de métodos e evidências científicas rígidas, consiste no padrão ouro de cuidado à saúde. (Ferreira, Silveira, Silva, Souza, \& Ruiz, 2016). A pesquisa em enfermagem oncológica é essencial para gerar a base de conhecimento que fundamenta a prática clínica, além de poder identificar o impacto do câncer e do tratamento na vida de pacientes e familiares. (Silveira, \& Zago, 2006).

Nesse contexto, foi proposta a presente revisão integrativa da literatura, com o objetivo identificar resultados de estudos primários que investigaram a FSQ em mulheres com câncer de mama, apresentando o estado atual do conhecimento, com vistas a aprofundar e 
oferecer melhor embasamento científico para a prática da assistência à esta população.

\section{Método}

Para o desenvolvimento de pesquisa na prática profissional de enfermagem, é importante ter consciência dos referenciais metodológicos, da definição clara e objetiva do objeto de estudo, para a seleção da metodologia mais adequada a ser aplicada. Para se obter respostas e resultados aos seus questionamentos, a enfermagem busca ampliar a produção de conhecimentos, por meio da realização de pesquisas. (Grittem, Méier, \& Zagonel, 2008).

Devido ao potencial para apresentar uma compreensão abrangente dos problemas relevantes para o cuidado em saúde e de incorporar diversas metodologias, permitindo aos revisores sintetizar resultados sem ferir a filiação epistemológica dos estudos empíricos incluídos, a revisão integrativa foi eleita como método para o alcance dos objetivos desse estudo. (Soares et al., 2014; Whittemore, \& Knafl, 2005). Este método tem o intuito de reduzir incertezas sobre práticas realizadas, facilitar o processo de tomada de decisão na prática profissional, o que resulta em um cuidado de saúde mais efetivo. (Mendes, Silveira, \& Galvão, 2008).

Uma revisão integrativa bem realizada exige os mesmos padrões de rigor, clareza e replicação utilizada nos estudos primários, para que seu produto possa trazer contribuições significativas para a ciência e para a prática clínica. A preservação deste padrão requer o uso de métodos que garantam a análise precisa, objetiva e completa do tema revisado, suporte e a provisão de todas as informações contidas nos estudos revisados, de modo a informar o leitor sem o sobrecarregar com informações desnecessárias. (Soares et al., 2014)

Este estudo foi realizado em seis etapas: identificação do tema e seleção da questão norteadora da pesquisa, estabelecimento dos critérios de inclusão e exclusão, identificação dos estudos préselecionados e selecionados, categorização dos estudos selecionados, análise e interpretação dos resultados, apresentação da revisão/síntese do conhecimento. (Botelho, Cunha, \& Macedo, 2011). Buscando responder ao objetivo do estudo, foi definida como pergunta norteadora: $O$ que tem sido investigado e publicado em meio científico sobre a fadiga secundária à quimioterapia em mulheres com câncer de mama? 
A amostra foi composta por artigos científicos nacionais e internacionais, desenvolvidos por profissionais de saúde, que investigaram a FSQ em mulheres com câncer de mama. O levantamento bibliográfico foi realizado nas seguintes bases de dados: US National Library of Medicine (PUBMED), U.S. National Library of Medicine (MEDLINE), Cumulative Index to Nursing and Allied Health Literature (CINAHL), base de dados da American Psychological Association (PsycINFO), Biblioteca Virtual em Saúde (BVS), Scientific Electronic Library Online (SCIELO), Literatura Latino-Americana e do Caribe em Ciências da Saúde (LILACS) e Scopus.

As buscas nas bases de dados foram realizadas durante os meses de janeiro a setembro de 2016, por meio do cruzamento dos Descritores em Ciências da Saúde (DECS): fatigue/fadiga, breast neoplasms/ neoplasias da mama, chemotherapy/ quimioterapia, nursing care/cuidado de enfermagem, interligados pelos operadores booleanos and, or. A base de dados PsycInfo possui seus próprios descritores, porém os termos corresponderam aos do
DECS. Estudos que abordaram a fadiga como reação adversa à quimioterapia, publicados no período de 2010 a 2016, nos idiomas inglês, português e espanhol, disponíveis na íntegra nas bases de dados eletrônicas em formato de artigos, foram incluídos.

Estabelecemos como critérios de exclusão: artigos em outros idiomas - que não português, inglês ou espanhol; artigos que descreviam fases de pesquisas e resultados relacionadas a medicamentos (estudos de fase); estudos que não abordassem a temática.

Por meio das buscas nas bases de dados, foram pré-selecionados 1181 artigos, os quais após a leitura dos títulos e resumos de estudos elegíveis, considerando os critérios de inclusão e exclusão, foi obtida uma amostra final de 12 estudos, conforme disposto na Figura 1. Artigos repetidos em diferentes bases de dados foram considerados uma única vez. 


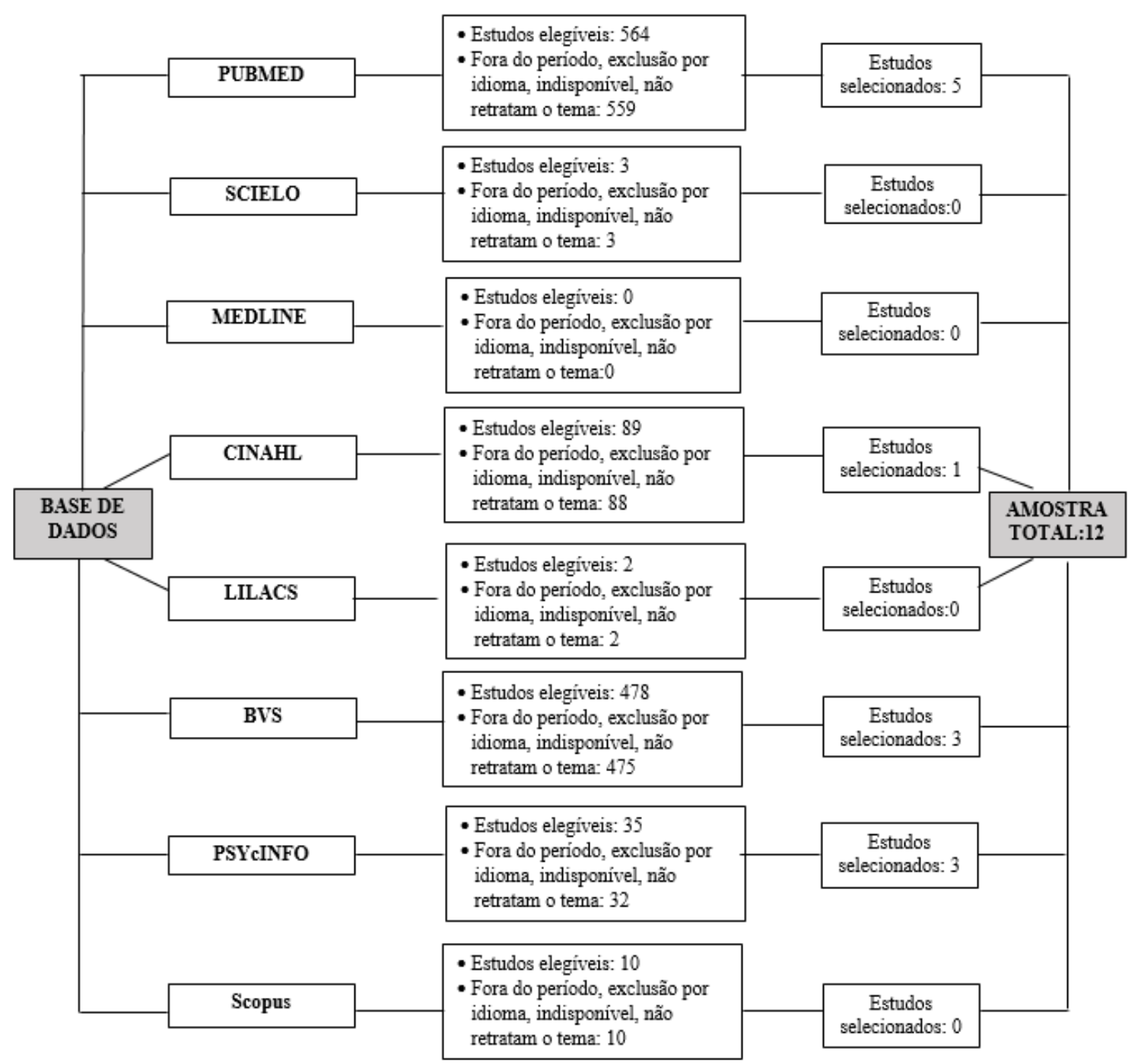

Figura 1 - Fluxograma da seleção dos estudos e motivos de exclusão.

A partir da leitura na íntegra dos estudos, realizada às cegas por pares Resultados buscando aumentar a acurácia e confiabilidade da avaliação - procedeu-se A caracterização dos estudos a caracterização dos estudos e coleta de incluídos - cuja identificação está disposta dados utilizando o instrumento proposto e elaborado por Ursi, e adaptado pelas de forma numérica - é apresentada no Figura 2.

autoras desta revisão. (Ursi, 2005). 


\begin{tabular}{|c|c|c|c|c|c|}
\hline Título dos artigos & $\begin{array}{l}\text { Base de } \\
\text { dados }\end{array}$ & Periódico & Autores & País & Ano \\
\hline $\begin{array}{l}\text { E1 - Effects of an 18-week } \\
\text { exercise programme started } \\
\text { early during breast cancer } \\
\text { treatment: a randomised } \\
\text { controlled trial. }\end{array}$ & Pubmed & BMC Medicine & $\begin{array}{l}\text { Travier N. et } \\
\text { al. }\end{array}$ & Noruega & 2015 \\
\hline $\begin{array}{l}\text { E2 - Women's perceptions of } \\
\text { home-based exercise } \\
\text { performed during adjuvant } \\
\text { chemotherapy for breast } \\
\text { cancer. }\end{array}$ & BVS & $\begin{array}{l}\text { European Journal } \\
\text { of Oncology } \\
\text { Nursing }\end{array}$ & $\begin{array}{l}\text { Ingram C., } \\
\text { Wessel J., \& } \\
\text { Courneya, K. } \\
\text { S. }\end{array}$ & Canadá & 2010 \\
\hline $\begin{array}{l}\text { E3 - Changes in and } \\
\text { predictors of severity of } \\
\text { fatigue in women with breast } \\
\text { cancer: a longitudinal study. }\end{array}$ & PSYcINFO & $\begin{array}{l}\text { International } \\
\text { Journal of } \\
\text { Nursing Studies }\end{array}$ & $\begin{array}{l}\text { Huang, H.P., } \\
\text { Chen, M.L., } \\
\text { Liang, J., \& } \\
\text { Miaskowski, } \\
\text { C. }\end{array}$ & Taiwan & 2014 \\
\hline $\begin{array}{l}\text { E4 - Ecological momentary } \\
\text { assessment of sleep, } \\
\text { symptoms, and mood during } \\
\text { chemotherapy for breast } \\
\text { cancer. }\end{array}$ & PSYcINFO & Psycho-Oncology & $\begin{array}{l}\text { Ratcliff, C.G., } \\
\text { Lam, C.Y., } \\
\text { Arun, B., } \\
\text { Valero, V., \& } \\
\text { Cohen, L. }\end{array}$ & EUA & 2014 \\
\hline $\begin{array}{l}\text { E5 - Epigenetic changes } \\
\text { associated with inflammation } \\
\text { in breast cancer patients } \\
\text { treated with chemotherapy. }\end{array}$ & PSYcINFO & $\begin{array}{l}\text { Brain, Behavior, } \\
\text { and Immunity }\end{array}$ & $\begin{array}{l}\text { Smith, A. K. et } \\
\text { al. }\end{array}$ & EUA & 2014 \\
\hline $\begin{array}{l}\text { E6 - Effects of Scheduled } \\
\text { Exercise on Cancer-Related } \\
\text { Fatigue in Women with Early } \\
\text { Breast Cancer. }\end{array}$ & Pubmed & $\begin{array}{l}\text { The Scientific } \\
\text { World Journal }\end{array}$ & $\begin{array}{l}\text { Husebo, } \\
\text { A.M.L., } \\
\text { Dyrstad, S.M., } \\
\text { Mjaaland, I., } \\
\text { Soreide, J.A., } \\
\text { \& Bru, E. }\end{array}$ & Noruega & 2014 \\
\hline $\begin{array}{l}\text { E7 - Fatigue and Sleep } \\
\text { Quality are Associated with }\end{array}$ & BVS & $\begin{array}{l}\text { NIH Public } \\
\text { Access }\end{array}$ & Liu, L. et al. & EUA & 2012 \\
\hline
\end{tabular}




\begin{tabular}{|c|c|c|c|c|c|}
\hline $\begin{array}{l}\text { Changes in Inflammatory } \\
\text { Markers in Breast Cancer } \\
\text { Patients Undergoing } \\
\text { Chemotherapy. }\end{array}$ & & & & & \\
\hline $\begin{array}{l}\text { E8 - Prevalência de } \\
\text { depressão e fadiga em um } \\
\text { grupo de mulheres com } \\
\text { câncer de mama. }\end{array}$ & BVS & $\begin{array}{l}\text { Revista } \\
\text { Eletrônica de } \\
\text { Enfermagem }\end{array}$ & $\begin{array}{l}\text { Panobianco, } \\
\text { M. S. et al. }\end{array}$ & Brasil & 2012 \\
\hline $\begin{array}{l}\text { E9 - Effect of Withania } \\
\text { somnifera (Ashwagandha) on } \\
\text { the development of } \\
\text { chemotherapy-induced } \\
\text { fatigue and quality of life in } \\
\text { breast cancer patients. }\end{array}$ & Pubmed & $\begin{array}{l}\text { Integrative } \\
\text { Cancer Therapies }\end{array}$ & $\begin{array}{l}\text { Biswal, B.M., } \\
\text { Sulaiman, } \\
\text { S.A., Ismail, } \\
\text { H.C., Zakaria, } \\
\text { H., \& Musa, } \\
\text { K.I. }\end{array}$ & Malásia & 2012 \\
\hline $\begin{array}{l}\text { E10 - Effects of a home- } \\
\text { based walking training } \\
\text { program on cardiorespiratory } \\
\text { fitness in breast cancer } \\
\text { patients receiving adjuvant } \\
\text { chemotherapy: a pilot study. }\end{array}$ & Pubmed & $\begin{array}{l}\text { European Journal } \\
\text { Physical and } \\
\text { Rehabilitation } \\
\text { Medicine }\end{array}$ & Vicent F. et al. & França & 2013 \\
\hline $\begin{array}{l}\text { E11 - Light Treatment } \\
\text { Prevents Fatigue in Women } \\
\text { Undergoing Chemotherapy } \\
\text { for Breast Cancer. }\end{array}$ & Pubmed & $\begin{array}{l}\text { Support Care } \\
\text { Cancer }\end{array}$ & $\begin{array}{l}\text { Ancoli-Israel } \\
\text { S. et al. }\end{array}$ & EUA & 2012 \\
\hline $\begin{array}{l}\text { E12 - Fatigue in Breast } \\
\text { Cancer Patients on Adjuvant } \\
\text { Treatment: Course and } \\
\text { Prevalence. }\end{array}$ & Cinahl & $\begin{array}{l}\text { Indian Journal of } \\
\text { Palliative Care }\end{array}$ & $\begin{array}{l}\text { Manir K. S. et } \\
\text { al. }\end{array}$ & Índia & 2012 \\
\hline
\end{tabular}

Figura 2 - Caracterização dos estudos. Uberlândia, MG, Brasil, 2016.

A amostra final de 12 estudos foi indexada nas seguintes bases de dados: cinco na Pubmed, três na BVS, três na PSYcINFO e um na Cinahl.

De acordo com as características identificadas, em relação à área profissional dos autores, sete estudos foram realizados por médicos e cinco por enfermeiros, os quais tiveram colaboração de outras áreas profissionais como nutrição, fisioterapia, educação física, psicologia e educação. As publicações 
originaram-se de diversos países, sendo quatro dos Estados Unidos da América (EUA), dois da Noruega, um do Brasil e os outros cinco estudos foram provenientes do Canadá, Taiwan, Malásia, França e Índia.

O inglês prevaleceu como idioma de publicação, sendo verificado em 11 estudos; apenas um artigo foi publicado em português. Apesar de a língua espanhola ser um dos idiomas estabelecidos como critério de inclusão, nenhum estudo neste idioma - relacionado à temática - foi selecionado. Em relação ao ano de publicação, cinco estudos foram publicados em 2012 e quatro estudos em 2014, os demais foram publicados nos anos de 2010, 2013 e 2015.

A seguir, na Figura 3, são apresentadas informações sobre o delineamento dos estudos - metodologia, método de coleta e análise dos dados objetivos e resultados dos estudos incluídos:

\begin{tabular}{|c|c|c|c|}
\hline & Delineamento & Objetivos & Resultados \\
\hline E1 & $\begin{array}{l}\text { Estudo quantitativo. } \\
\text { Delineamento } \\
\text { experimental. Coleta de } \\
\text { dados: escalas. Análise dos } \\
\text { dados: Análise de } \\
\text { sensibilidade, análise por } \\
\text { protocolo. }\end{array}$ & $\begin{array}{l}\text { Avaliar o efeito de } 18 \\
\text { semanas de exercício } \\
\text { aeróbico e de resistência } \\
\text { diário na diminuição da } \\
\text { fadiga. }\end{array}$ & $\begin{array}{l}\text { A intervenção mostrou efeitos } \\
\text { benéficos na fadiga física, capacidade } \\
\text { cardiorrespiratória e força muscular no } \\
\text { grupo de intervenção em } 18 \text { semanas. }\end{array}$ \\
\hline E2 & $\begin{array}{l}\text { Estudo qualitativo. } \\
\text { Referencial teórico não } \\
\text { mencionado. Coleta de } \\
\text { dados: entrevista } \\
\text { telefônica. Análise de } \\
\text { dados: análise de } \\
\text { conteúdo. }\end{array}$ & $\begin{array}{l}\text { Descrever as percepções dos } \\
\text { pacientes de um programa de } \\
\text { exercícios, estruturado, } \\
\text { domiciliar durante a } \\
\text { quimioterapia adjuvante para } \\
\text { o câncer de mama. }\end{array}$ & $\begin{array}{l}\text { Os desafios mais comuns foram os } \\
\text { efeitos colaterais decorrentes da } \\
\text { quimioterapia, sendo que a fadiga foi a } \\
\text { mais frequente. Os benefícios } \\
\text { emocionais e físicos do exercício } \\
\text { foram descritos como fontes de } \\
\text { motivação. }\end{array}$ \\
\hline E3 & $\begin{array}{l}\text { Estudo quantitativo. } \\
\text { Delineamento não } \\
\text { experimental. Coleta de } \\
\text { dados: escalas e } \\
\text { questionário. Análise de } \\
\text { dados: Análise multinível. }\end{array}$ & $\begin{array}{l}\text { 1) Explorar a trajetória da } \\
\text { fadiga } 1 \text { ano pós cirurgia em } \\
\text { mulheres com câncer de } \\
\text { mama. } \\
\text { 2) Determinar se a fadiga } \\
\text { flutua com os níveis dos } \\
\text { sintomas depressivos. }\end{array}$ & $\begin{array}{l}\text { A severidade da fadiga atingiu seu } \\
\text { pico no } 2^{\circ} \text { mês após a cirurgia, e com } \\
\text { queda mais acelerada no } 5^{\circ} \text { mês. No } 6^{\circ} \\
\text { mês a fadiga já havia voltado ao nível } \\
\text { pré-operatório, e } 12 \text { meses depois era } \\
\text { menor que os níveis pré-operatórios. } \\
\text { Pacientes que tinham altos níveis de }\end{array}$ \\
\hline
\end{tabular}


FADIGA SECUNDÁRIA À QUIMIOTERAPIA EM MULHERES COM CÂNCER DE MAMA: REVISÃO INTEGRATIVA DE LITERATURA

\begin{tabular}{|c|c|c|c|}
\hline & & $\begin{array}{l}\text { 3) Identificar os fatores que } \\
\text { influenciam a mudança da } \\
\text { fadiga após controlar os } \\
\text { sintomas depressivos. }\end{array}$ & $\begin{array}{l}\text { sintomas depressivos tenderam a ter } \\
\text { níveis maiores de fadiga. }\end{array}$ \\
\hline E4 & $\begin{array}{l}\text { Estudo quantitativo. } \\
\text { Delineamento não- } \\
\text { experimental. Coleta de } \\
\text { dados: escalas e } \\
\text { Avaliação Momentânea } \\
\text { Ecológica. Análise de } \\
\text { dados: Software G Power } \\
\text { e método de Bonferroni. }\end{array}$ & $\begin{array}{l}\text { Examinar a relação entre o } \\
\text { sono e os sintomas } \\
\text { relacionados ao câncer } \\
\text { durante o ciclo de QT. }\end{array}$ & $\begin{array}{l}\text { As mulheres relataram gravidade } \\
\text { relativamente baixa dos sintomas, com } \\
\text { a fadiga recebendo a maior } \\
\text { classificação de gravidade. As } \\
\text { mulheres que relataram distúrbios do } \\
\text { sono tenderam a apresentar maior } \\
\text { fadiga geral. Existe a tendência de que } \\
\text { distúrbios do sono antes da QT podem } \\
\text { ser associados com maior fadiga e } \\
\text { humor negativo durante a QT. }\end{array}$ \\
\hline E5 & $\begin{array}{l}\text { Estudo quantitativo. } \\
\text { Delineamento não } \\
\text { experimental. Coleta de } \\
\text { dados: questionário, coleta } \\
\text { de material biológico. } \\
\text { Análise de dados: Análise } \\
\text { de covariância. }\end{array}$ & $\begin{array}{l}\text { Identificar diferenças } \\
\text { epigenéticas relacionadas à } \\
\text { quimioterapia, associadas ao } \\
\text { aumento da inflamação e/ou } \\
\text { à fadiga relacionada ao } \\
\text { câncer. }\end{array}$ & $\begin{array}{l}\text { Pacientes em QT apresentaram no } \\
\text { início maior concentração plasmática } \\
\text { de receptor solúvel de fator de necrose } \\
\text { tumoral (sTNFR2) e leve aumento da } \\
\text { interleucina } 6 \text { (IL-6) no plasma; } \\
\text { também tiveram escores } \\
\text { significantemente maiores de fadiga. } \\
\text { As concentrações de sTNFR2 e a IL-6 } \\
\text { foram positivamente correlacionadas } \\
\text { com a fadiga. }\end{array}$ \\
\hline E6 & $\begin{array}{l}\text { Estudo quantitativo. } \\
\text { Delineamento } \\
\text { experimental. Coleta de } \\
\text { dados: Escalas, teste de } \\
\text { caminhada e exercício } \\
\text { diário. Análise de dados: } \\
\text { Análise de variância e } \\
\text { tabulações cruzadas, } \\
\text { análises descritivas, testes } \\
\text { de confiabilidade, } \\
\text { ANOVA. }\end{array}$ & $\begin{array}{l}\text { Comparar efeitos de um } \\
\text { exercício programado } \\
\text { domiciliar para intervenção } \\
\text { na fadiga relacionada ao } \\
\text { câncer, aptidão física e nível } \\
\text { de atividade, em comparação } \\
\text { com o exercício físico } \\
\text { regular. }\end{array}$ & $\begin{array}{l}\text { Os níveis de fadiga aumentaram } \\
\text { significativamente desde o início até o } \\
\text { fim da quimioterapia para toda a } \\
\text { amostra. Não foram encontradas } \\
\text { diferenças significativas na trajetória } \\
\text { de fadiga entre os grupos. }\end{array}$ \\
\hline E7 & $\begin{array}{l}\text { Estudo quantitativo. } \\
\text { Delineamento não } \\
\text { experimental. Coleta de }\end{array}$ & $\begin{array}{l}\text { Identificar a relação entre } \\
\text { marcadores biológicos e } \\
\text { fadiga e distúrbios do sono }\end{array}$ & $\begin{array}{l}\text { Durante a quimioterapia, a fadiga era } \\
\text { pior do que pré-quimioterapia, } \\
\text { enquanto a má qualidade do sono }\end{array}$ \\
\hline
\end{tabular}




\begin{tabular}{|c|c|c|c|}
\hline & $\begin{array}{l}\text { dados: questionários, } \\
\text { actígrafo, coleta de } \\
\text { material biológico. Análise } \\
\text { de dados: Análise de } \\
\text { modelo misto. }\end{array}$ & relacionados ao câncer. & $\begin{array}{l}\text { permaneceu. Mudanças na fadiga e na } \\
\text { qualidade do sono foram } \\
\text { significativamente associados a } \\
\text { mudanças de níveis circulantes de IL- } \\
\text { 6. Não houve associação significativa } \\
\text { entre alterações na fadiga ou na } \\
\text { qualidade do sono e mudanças nos } \\
\text { marcadores inflamatórios. }\end{array}$ \\
\hline E8 & $\begin{array}{l}\text { Estudo quantitativo. } \\
\text { Delineamento não- } \\
\text { experimental. Coleta de } \\
\text { dados: Escalas e } \\
\text { questionários. Análise de } \\
\text { dados: conforme } \\
\text { orientação dos autores. }\end{array}$ & $\begin{array}{l}\text { Identificar e avaliar a } \\
\text { ocorrência de sintomas } \\
\text { depressivos e de fadiga entre } \\
\text { mulheres com até um ano de } \\
\text { tratamento com radioterapia } \\
\text { e/ou quimioterapia para o } \\
\text { câncer de mama. }\end{array}$ & $\begin{array}{l}\text { Em relação à fadiga, foi possível } \\
\text { identificar sintomas de mal-estar geral } \\
\text { como, cansaço nas pernas, cansaço no } \\
\text { corpo todo, desejo de deitar-se durante } \\
\text { o dia e torpor. } \\
\text { Quanto à ocorrência da fadiga, as } \\
\text { mulheres atribuíram aos sintomas de } \\
\text { cansaço à cirurgia, à radioterapia, à } \\
\text { quimioterapia e à preocupação. } \\
\text { As estratégias utilizadas para aliviar } \\
\text { tais sintomas foram: sentar ou deitar } \\
\text { em algum período do dia caminhar ou } \\
\text { fazer algum tipo de exercício. } \\
\text { A maioria das participantes manifestou } \\
\text { sintomas depressivos entre leve e } \\
\text { moderado. }\end{array}$ \\
\hline E9 & $\begin{array}{l}\text { Estudo quantitativo. } \\
\text { Delineamento } \\
\text { Experimental. Coleta de } \\
\text { dados: Escalas. } \\
\text { Análise de dados: } \\
\text { Software SPSS (versão 18, } \\
\text { IBM); medidas repetidas } \\
\text { ANCOVA; método de } \\
\text { Kaplan-Meier. }\end{array}$ & $\begin{array}{l}\text { Propor o uso de extrato de } \\
\text { Withania somnifera (WS) } \\
\text { para o tratamento da fadiga } \\
\text { induzida pela quimioterapia } \\
\text { e da preservação da } \\
\text { qualidade de vida (QV) entre } \\
\text { pacientes com câncer de } \\
\text { mama. }\end{array}$ & $\begin{array}{l}\text { A fadiga foi maior no grupo controle } \\
\text { em comparação com os grupos } \\
\text { receberam WS. Withania somnifera } \\
\text { pode ser um agente potencial para a } \\
\text { melhoria da qualidade de vida e } \\
\text { melhora da fadiga. }\end{array}$ \\
\hline E10 & $\begin{array}{l}\text { Estudo quantitativo. } \\
\text { Delineamento } \\
\text { experimental. Coleta de } \\
\text { dados: exames }\end{array}$ & $\begin{array}{l}\text { Examinar os efeitos de uma } \\
\text { abordagem individualizada } \\
\text { de treinamento de caminhada } \\
\text { domiciliar, avaliando a }\end{array}$ & $\begin{array}{l}\text { O programa de treinamento de } \\
\text { caminhada em domicílio teve um } \\
\text { efeito benéfico no desempenho } \\
\text { cardiorrespiratório. Para pacientes com }\end{array}$ \\
\hline
\end{tabular}




\begin{tabular}{|c|c|c|c|}
\hline & $\begin{array}{l}\text { laboratoriais, escala e } \\
\text { questionário. Analise de } \\
\text { dados: Teste de T Student } \\
\text { e teste de Wilcoxon. }\end{array}$ & $\begin{array}{l}\text { aptidão aeróbica e pico de } \\
\text { VO2, e os efeitos do } \\
\text { treinamento aeróbio sobre a } \\
\text { fadiga e outros parâmetros } \\
\text { cardiopulmonares durante a } \\
\text { quimioterapia em pacientes } \\
\text { que receberam terapia } \\
\text { adjuvante para o câncer de } \\
\text { mama em estágio inicial. }\end{array}$ & $\begin{array}{l}\text { taxa de adesão }<80 \% \text {, mudanças } \\
\text { significativas na capacidade de } \\
\text { exercício ou nos resultados da função } \\
\text { pulmonar foram observadas, alterações } \\
\text { na escala de fadiga não foram } \\
\text { estatisticamente significativas. } \\
\text { Nenhuma relação foi encontrada entre } \\
\text { escore de fadiga e atividade física. }\end{array}$ \\
\hline E11 & $\begin{array}{l}\text { Estudo quantitativo. } \\
\text { Delineamento } \\
\text { experimental. Coleta de } \\
\text { dados: actígrafos e } \\
\text { questionário. Análise de } \\
\text { dados: Teste t e teste exato } \\
\text { de Fisher. }\end{array}$ & $\begin{array}{l}\text { Testar a hipótese de que o } \\
\text { aumento da luz brilhante } \\
\text { matutina, em comparação } \\
\text { com a exposição à luz fraca, } \\
\text { resultaria em menos fadiga } \\
\text { durante a quimioterapia. }\end{array}$ & $\begin{array}{l}\text { O tratamento da luz brilhante da } \\
\text { manhã pode impedir a fadiga geral de } \\
\text { piorar durante a quimioterapia. O } \\
\text { grupo que recebia luz branca brilhante } \\
\text { apresentava melhora na subescala } \\
\text { emocional, enquanto que o grupo de } \\
\text { luz vermelha fraca não apresentava } \\
\text { alteração e menor vigor. }\end{array}$ \\
\hline E12 & $\begin{array}{l}\text { Estudo quantitativo. } \\
\text { Delineamento não } \\
\text { experimental. Coleta de } \\
\text { dados: questionário. } \\
\text { Análise de regressão } \\
\text { múltipla. }\end{array}$ & $\begin{array}{l}\text { Quantificar a prevalência, } \\
\text { curso e grau de fadiga em } \\
\text { pacientes com câncer de } \\
\text { mama em tratamento } \\
\text { adjuvante; efeito das } \\
\text { modalidades de tratamento } \\
\text { (quimioterapia e/ou } \\
\text { radioterapia) sobre o mesmo } \\
\text { e cofatores que influenciam } \\
\text { o cenário. }\end{array}$ & $\begin{array}{l}64 \text { pacientes ( } 83 \%) \text { apresentaram } \\
\text { fadiga. } 72(91 \%) \text { apresentaram fadiga } \\
\text { durante a quimioterapia. A prevalência } \\
\text { de fadiga aumentou significativamente } \\
\text { a partir da segunda avaliação; os } \\
\text { escores foram aumentados } \\
\text { significativamente do ciclo um para o } \\
\text { ciclo dois, mas do ciclo dois ao ciclo } \\
\text { seis as pontuações diminuíram } \\
\text { significativamente. }\end{array}$ \\
\hline
\end{tabular}

Figura 3 - Delineamento, objetivos e resultados dos estudos. Uberlândia, MG, Brasil, 2016.

No que diz respeito à metodologia utilizada, apenas o estudo E2 utilizou a metodologia qualitativa, os demais utilizaram a metodologia quantitativa. Em relação ao delineamento dos estudos, seis (E3, E4, E5, E7, E8, E12) utilizaram o delineamento não experimental e cinco (E1, E6, E9, E10, E11) o delineamento experimental. No estudo E2, com abordagem metodológica qualitativa, não constava o referencial teórico utilizado.

Os recursos mais utilizados para coleta de dados foram questionários (E3, E5, E7, E8, E10, E11, E12) e escalas (E1, E4, E6, E9). Em alguns casos estes recursos foram utilizados de forma 
conjunta e, em outros, foram acrescidos de métodos específicos, estabelecidos pelos autores - como coleta de material biológico; uso do actígrafo, que é um equipamento utilizado para diagnóstico, pesquisa e estudo do ciclo sono-vigília e seus ritmos circadianos - dentre outros. Nestes estudos foram realizadas análises estatísticas dos dados coletados.

Para coleta de dados do estudo E2 foram realizadas entrevistas telefônicas, as quais posteriormente foram submetidas à análise de conteúdo.

Os objetivos de todos os estudos foram apresentados de forma clara e de fácil entendimento. Em geral os estudos objetivaram avaliar intervenções para melhora da FSQ, verificar a relação da fadiga com outros sintomas decorrentes do tratamento, verificar a ocorrência da FSQ, bem como investigar alterações epigenéticas relacionadas à FSQ.

Por meio da análise dos resultados dos estudos foram identificados temas e subtemas que se destacaram pela similaridade e relevância com que foram descritos nos estudos, o que permitiu sintetizar e integrar os achados a respeito da FSQ em mulheres com câncer de mama, conforme disposto na Figura 4.

\section{Temas/ subtemas}

Artigos

1. Impacto da prática de exercícios físicos sobre a FSQ E1, E2, E6, E10

2. Relação entre a fadiga e outros sintomas decorrentes da quimioterapia E3, E4, E8

- Fadiga e depressão E3, E8

- Fadiga e distúrbios do sono E4

3. Alterações epigenéticas associadas à FSQ E5, E7

4. Utilização de medicina complementar e alternativa para tratamento da FSQ E9, E11

5. Trajetória da fadiga durante a quimioterapia para câncer de mama E3, E8, E12

Figura 4 - Temas e subtemas identificados na análise dos estudos. Uberlândia, MG, Brasil, 2016.

A distribuição dos temas identificados foi: tema um, presente em quatro dos 12 artigos (33\%); temas dois e cinco, abordados em três artigos (25\%); e os temas três e quatro abordados em dois estudos (16\%).

\section{Em relação ao tema "Impacto da} prática de exercícios físicos sobre a FSQ”, dos quatro estudos que avaliaram o impacto da prática de exercícios físicos sobre a FSQ, três (E1, E6, E10) apresentaram focos similares e um (E2) apresentou foco diferente dos demais. Os 
estudos E1, E6, E10 avaliaram os efeitos de programas de exercícios em pacientes com câncer de mama em QT. O estudo E2, devido sua metodologia qualitativa, buscou descrever as percepções das pacientes a respeito de um programa de exercícios.

Apenas o estudo E1, conseguiu confirmar a hipótese de que a prática de exercícios físicos diários é benéfica para controle da fadiga. Já nos estudos E6 e E10 não foram encontradas relações estatisticamente significantes entre a fadiga e a atividade física. No estudo E2, a FSQ apresentou-se como um desafio para a prática de exercícios físicos.

O tema "Relação entre a fadiga e outros sintomas decorrentes da quimioterapia", trata dos resultados de três estudos que exploraram a relação da FSQ com outros sintomas decorrentes dessa modalidade de tratamento. Os estudos E3 e E8 exploraram a relação entre a fadiga e sintomas depressivos; sendo que no estudo E3 foi evidenciado que pacientes com altos níveis de sintomas depressivos tenderam a ter mais fadiga; e no estudo E8 foi observado que a maioria das pacientes com queixas de fadiga manifestaram sintomas depressivos leves ou moderados.

O estudo E4 examinou a relação entre a fadiga e o sono, no qual foi observado que existe a tendência de que distúrbios do sono antes da QT podem ser associados com maior fadiga durante a QT. As participantes que relataram distúrbios do sono tenderam a apresentar maior fadiga.

No tema "Alterações epigenéticas associadas à FSQ", as concentrações de receptor solúvel de fator de necrose tumoral (sTNFR2) - avaliadas no estudo E5 - e de interleucina 6 (IL-6) - avaliadas nos estudos E5 e E7 - foram significativamente associados com a FSQ. Dessa forma, quanto maior a concentração plasmática desses marcadores biológicos, maiores serão os escores de fadiga.

$\mathrm{O}$ tema "Utilização de medicina complementar e alternativa para tratamento da FSQ", destaca que foram realizadas intervenções com práticas da medicina complementar e alternativa (MCA) com o objetivo de diminuir a FSQ. No estudo E9 foi proposto o uso de um fitoterápico e no estudo E11 foi realizada exposição à luz branca matutina.

Foi evidenciado que a utilização do fitoterápico proposto pode ser um agente potencial para a melhora da fadiga; já o tratamento com exposição à luz branca matutina pode impedir a piora da fadiga durante a QT.

\section{$\mathrm{O}$ tema "Trajetória da fadiga} durante a quimioterapia para câncer de mama", traz que a severidade da fadiga é maior durante a QT, como demonstrado no 
estudo E3. Alguns sintomas podem ser atribuídos à fadiga como: cansaço nas pernas e no corpo todo, desejo de deitar-se durante o dia e sonolência; sendo que sua ocorrência é relacionada à quimioterapia (E8). Há evidências (E12) de aumento significativo da fadiga do primeiro ciclo quimioterápico para o segundo, sendo que do segundo ao sexto ciclo essa prevalência tende a diminuir.

\section{Discussão}

Foram encontrados poucos estudos nos anos de 2010, 2013 e 2015; e no ano de 2016 não foi encontrado nenhum artigo que atendesse aos critérios de inclusão deste estudo, o que evidencia a necessidade de novas publicações a respeito deste tema.

Dentre os artigos incluídos, apenas um utilizou a metodologia qualitativa, tal fato traz à tona a carência de estudos com esta abordagem metodológica, no sentido de melhor compreender o processo de adoecimento e tratamento na perspectiva do sujeito, objetivando uma visão ampliada e integral dos problemas de saúde.

Da mesma forma, para efeito de discussão desta temática, foi identificado somente um estudo qualitativo, que investigou sobre a percepção das mulheres com câncer de mama em relação às reações adversas relacionadas à hormonioterapia e à QT, dentre elas a fadiga. Estas mulheres citaram a necessidade de maior atenção às suas necessidades. (Jodar, Jacquin, \& Vallée, 2016). Isto reforça que o profissional de saúde deve valorizar os relatos das pacientes e utilizar a escuta como importante ferramenta para uma assistência integral e de qualidade.

Dentre as formas de manejo da fadiga, a prática de exercícios físicos em pacientes com câncer de mama vem sendo investigada de forma crescente; a literatura traz que estudos realizados nos anos de 1999, 2000, 2001 e 2002 comprovaram a redução da fadiga com esta intervenção. (Bonassa, \& Oliveira, 2012). Isto mostra o interesse dos pesquisadores em proporcionar conforto aos pacientes oncológicos com práticas acessíveis.

Nesta revisão foi encontrado um resultado relevante no estudo $\mathrm{E} 1$, ao identificar que um programa de 18 semanas de exercício aeróbico e de resistência em mulheres com câncer de mama em QT trouxe resultados positivos no manejo da fadiga, resistência cardiorrespiratória e força muscular. De forma semelhante, um estudo realizado identificou que um programa de 12 semanas de caminhada foi eficiente na redução da fadiga para a mesma 
população. (ABCP, 2010). O que também foi evidenciado por duas revisões, sendo uma meta análise que identificou efeitos benéficos do exercício físico sobre a dimensão física da fadiga durante $o$ tratamento adjuvante para o câncer de mama; e uma revisão sistemática e meta análise que constatou melhora na fadiga de pacientes com câncer de mama e de próstata que realizaram exercício físico, e que considerou esta prática uma intervenção segura no manejo da fadiga para pacientes com câncer em tratamento. (Meneses-Echávez et al., 2015).

Além da prática de exercícios físicos ser considerada uma intervenção para a fadiga durante o tratamento, foi identificada a sua indicação para o manejo da fadiga após o tratamento não só para pacientes com câncer de mama, mas também para o manejo da fadiga em adultos sobreviventes de câncer. Uma diretriz publicada pela Sociedade Americana de Oncologia Clínica (ASCO), orienta que a prática de exercícios físicos adequados em sobreviventes de câncer pode reduzir a FRC após o tratamento. Desta forma, recomenda o incentivo à prática de um nível moderado de atividade física, como 150 minutos por semana de exercício aeróbico, incluindo caminhada, ciclismo ou natação, associado a 2-3 sessões de treinamento de força, desde que não seja contraindicado ao paciente. (Bower et al., 2014).

Entretanto, nos estudos E6 e E10, não foram identificadas diferenças significativas em relação à prática de exercícios físicos para a fadiga. Assim como um estudo, realizado com mulheres com câncer de mama durante a QT, que não encontrou resultados significativos para o exercício físico na atenuação da fadiga e promoção da qualidade de vida. (Al-Majid, Wilson, Rakovski, \& Coburn, 2015).

O estudo E2 identificou que apesar do desafio, a prática de exercícios físicos foi considerada benéfica pelas mulheres durante $o$ tratamento adjuvante para $o$ câncer de mama. Os desafios foram superados com adaptação à rotina, motivação interna e apoio externo. $\mathrm{O}$ estudo mostra que a compreensão da doença e de seu tratamento, reforçada pelos especialistas, auxiliou estas mulheres no desenvolvimento de estratégias para manterem o programa de exercícios, o que demonstra o importante papel dos profissionais que atuam na assistência junto à estas pacientes.

Diante dos resultados encontrados, nota-se a necessidade de novas pesquisas que possam contribuir na definição do melhor tipo de exercício a ser indicado, considerando inclusive sua intensidade e 
tempo de duração para o tratamento e/ou redução da FSQ.

No que diz respeito à relação entre a fadiga e outros sintomas decorrentes da quimioterapia, um estudo mostrou resultado inverso ao estudo E3, onde pacientes com maiores níveis de fadiga apresentaram humor mais deprimido, quando comparadas às que tiveram baixo nível de sintomas depressivos; porém, as pacientes que apresentaram fadiga transitória, tiveram alteração de humor semelhante às que apresentaram alto nível de fadiga. (Junghaenel, Cohen, Schneider, Neerukonda, \& Broderick, 2015).

Vale ressaltar, que foi encontrada associação entre fadiga e depressão em pacientes com câncer de mama de um a seis meses após a conclusão da QT (Levkovich, Cohen, Pollack, Drumea, \& Fried, 2015), o que demonstra a importância da avaliação e alívio destas reações durante e após o tratamento.

A literatura reforça que pacientes com câncer apresentam alteração do padrão de sono. Em geral o sono é interrompido diversas vezes à noite, sendo freqüentes os momentos de cochilo durante o dia e altos níveis de fadiga. Isto é considerado comum em pacientes que realizam pouca atividade durante o dia. (Bonassa, \& Oliveira, 2012).
Em relação à interleucina 6 (IL-6), um estudo que não identificou relação entre IL-6 e FSQ, encontrou associação entre os níveis de fator de necrose tumoral alfa $(\mathrm{TNF}-\alpha)$ e a fadiga em pacientes com câncer de mama. (Cruz, Lerner, Munhoz, Fonseca, Cubero, \& Giglio, 2013). A IL-6 e o TNF- $\alpha$ são citocinas pró-inflamatórias, ou seja, que podem aumentar a resposta inflamatória em caso de infecção ou dano tecidual, por exemplo. (Oliveira, Sakata, Issy, Gerola, \& Salomão, 2011). Considerando a ação destas citocinas inflamatórias, tem sido proposta a relação entre fadiga e resposta inflamatória aumentada.

No que se refere à utilização da MCA para tratamento da FSQ, a literatura demonstra a redução da fadiga em pacientes com câncer de mama em QT com a prática de ioga, considerada uma intervenção corpo-mente. (Wang, Wang, Jiang, \& Zeng, 2014; Taso et al., 2014). Outras terapias complementares mostraram benefícios para a melhora da fadiga, como a eletroacupuntura para pacientes com câncer de mama em tratamento com inibidor de aromatase (Mao et al., 2014); o uso do extrato seco de Paullinia cupana (guaraná) para pacientes com outros tipos de tumores sólidos - neste estudo o autor reforça que este fitoterápico foi citado 
previamente como eficaz para o tratamento da fadiga em pacientes com câncer de mama (Giglio et al., 2013), o que também foi apresentado por um estudo em um encontro da ASCO, onde uma dosagem padronizada do extrato de guaraná auxiliou na diminuição do cansaço em mulheres durante a QT. (Bonassa, \& Oliveira, 2012).

Apesar de não existir um protocolo padrão a respeito do uso da MCA para o tratamento da FSQ, nota-se o interesse dos autores em investigar sobre esta temática e oferecer alívio a estas pacientes.

Corroborando com os resultados desta revisão em relação à trajetória da fadiga, há evidências que a fadiga se intensifica ao longo da quimioterapia em pacientes com câncer de mama (Guimarães, \& Anjos, 2012); bem como em relação aos sintomas apresentados pelos pacientes com FSQ, os quais relatam se sentir fracos, esgotados, cansados, pesados e sem energia. (National Cancer Institute, 2015).

Neste estudo foi citado sobre o aumento significativo da fadiga do primeiro para o segundo ciclo de quimioterapia. De forma semelhante, para outro autor, a fadiga ocorre de forma cumulativa entre os ciclos; em seu estudo mais da metade das participantes apresentaram piora da fadiga do primeiro para o segundo ciclo quimioterápico. (Cruz et al., 2013).

Vale ressaltar, que a fadiga é subjetiva. (Bonassa, \& Oliveira, 2012). A FSQ pode ser descrita e ter sua intensidade classificada de formas distintas pelos pacientes ao longo do tratamento, o que torna importante sua avaliação e intervenção durante todos os ciclos quimioterápicos e também no póstratamento. Vale ainda destacar a importância da valorização por parte do profissional às diversas formas que os pacientes descrevem a FSQ.

\section{Conclusão}

Esta revisão integrativa possibilitou sintetizar os achados de diferentes pesquisas a respeito da fadiga secundária à quimioterapia, trazendo contribuições significativas para a construção do conhecimento e para a prática assistencial.

A fadiga é um evento adverso muito frequente e comum entre pacientes com câncer de mama em QT e tem sido investigada de maneira crescente pelos pesquisadores. É notório o interesse envolvendo os diversos temas descritos nesta revisão, o que proporcionou melhor compreensão do cenário da FSQ em pacientes com câncer de mama. 
A FSQ pode estar relacionada a outros efeitos adversos durante a QT. Foi evidenciado que a intensidade deste evento adverso pode aumentar durante esta modalidade terapêutica, causando sensações de desconforto à estas pacientes. Em relação ao tratamento da FSQ, ainda não há um consenso a ser seguido pelos profissionais de saúde, mas existem possibilidades para proporcionar alívio, por meio da prática de atividades físicas conforme tolerância e indicação médica além de recursos terapêuticos não farmacológicos, como a terapia corpomente e o uso de fitoterápicos.
Frente ao exposto, considera-se importante a realização de estudos futuros para a definição de condutas mais efetivas para o tratamento da FSQ, queixa que nem sempre é valorizada pelos profissionais envolvidos no tratamento do câncer, mas que é potencialmente capaz de causar prejuízos que afetam diversas dimensões da vida desta população.

\section{Referências}

Al-Majid, S., Wilson, L.D., Rakovski, C., \& Coburn, J.W. (2015) Effects of exercise on biobehavioral outcomes of fatigue during cancer treatment: results of a feasibility study. Biological Research for Nursing, 17(1), 40-48. https://doi.org/10.1177/1099800414523489

Ancoli-Israel, S., Rissling, M., Neikrug, A., Trofimenko, V., Natarajan, L., Parker, B.A., Lawton, S., Desan, P., \& Liu, L. (2012). Light treatment prevents fatigue in women undergoing chemotherapy for breast cancer. Supportive Care in Cancer, 20(6), 12111219. https://doi.org/10.1007/s00520-011-1203-z

Associação Brasileira de Cuidados Paliativos (2010). Consenso Brasileiro de Fadiga. Revista Brasileira de Cuidados Paliativos. São Paulo: ABCP.

Biswal, B.M., Sulaiman, S.A., Ismail, H.C., Zakaria, H., \& Musa, K.I. (2012). Effect of Withania somnifera (Ashwagandha) on the development of chemotherapy-induced fatigue and quality of life in breast cancer patients. Integrative Cancer Therapies, 12(4), 312-322. https://doi.org/10.1177/1534735412464551

Bonassa, E.M.A., \& Oliveira, A.C. (2012) Fadiga. In E. M. A. Bonassa, \& M. I. R. Gato (4 ${ }^{\mathrm{a}}$ ed.), Terapêutica oncológica para enfermeiros e farmacêuticos. São Paulo: Atheneu. 
Botelho, L.L.R., Cunha, C.C.A., Macedo, M. (2011). O método da revisão integrativa nos estudos organizacionais. Gestão e Sociedade, 5(11), 121-136. https://doi.org/10.21171/ges.v5i11.1220

Bower, J.E., Bak, K., Berger, A., Breitbart, W., Escalante, C.P., Ganz, P.A., Schnipper, H.H., Lacchetti, C., Ligibel, J.A., Lyman, G.H., Ogaily, M.S., Pirl, W.F., \& Jacobsen, P.B. (2014). Screening, Assessment, and Management of Fatigue in Adult Survivors of Cancer: An American Society of Clinical Oncology Clinical Practice Guideline Adaptation. Journal of Clinical Oncology, 32(17), 1840-1850. https://doi.org/10.1200/JCO.2013.53.4495

Brasil. Ministério da Saúde. Instituto Nacional de Câncer José Alencar Gomes da Silva (2015). Estimativa 2016: incidência de câncer no Brasil. Rio de Janeiro: INCA.

Carr, A.C., Vissers, M.C.M., \& Cook, J.S. (2014). The effect of intravenous vitamin C on cancer- and chemotherapy-related fatigue and quality of life. Frontiers in Oncology, 4(283), 1-7. https://doi.org/10.3389/fonc.2014.00283

Cruz, F.M., Lerner, T.G., Munhoz, B., Fonseca, F., Cubero, D., Giglio, A.D. (2013) Quimioterapia induz fadiga e aumenta TNF- $\alpha$ em pacientes com câncer de mama. Revista Brasileira de Oncologia Clínica, 9(32), 52-54.

Curt, G.A., Breitbart, W., Cella, D., Groopman, J.E., Horning, S.J., Itri, L.M., Johnson, D.H., Miaskowski, C., Scherr, S.L., Portenoy, R.K., \& Vogelzang, N.J. (2000). Impact of cancer-related fatigue on the lives of patients: new findings from the Fatigue Coalition. The Oncologist. 2000; 5(5), 353-360. https://doi.org/10.1634/theoncologist.55-353

Diettrich, S.H.C., Miranda, C.R.R., Honer, M.R., Furtado E.R., \& Correa Filho, R.A.C. (2006). Efeitos de um programa de caminhada sobre os níveis de fadiga em pacientes com câncer de mama. Revista Brasileira de Ciência e Movimento, 14(1), 7-12.

Ferreira, M.B.G., Silveira, C.F., Silva, S.R.D., Souza D.J.D., \& Ruiz, M.T. (2016) Nursing care for women with pre-eclampsia and/or eclampsia: integrative review. Revista $d a$ Escola de Enfermagem da USP, 50(2), 324-334. https://doi.org/10.1590/S0080623420160000200020

Giglio, A.B.D., Cubero, D.D.I.G., Lerner, T.G., Guariento, R.T., Azevedo, R.G.S., Paiva, H., Goldman, C., Carelli, B., Cruz, F.M., Schindler, F., Pianowski, L., de Matos, L.L., \& del Giglio, A. (2013). Purified dry extract of Paullinia cupana (guaraná) (PC-18) for chemotherapy-related fatigue in patients with solid tumors: an early discontinuation study. Journal of Dietary Supplements, 10(4), 325-334. https://doi.org/10.3109/19390211.2013.830676

Grittem, L., Méier, M.J., \& Zagonel, I.P.S. (2008) Pesquisa-ação: uma alternativa metodológica para pesquisa em enfermagem. Texto \& Contexto - Enfermagem, 17(4), 765-770. https://doi.org/10.1590/S0104-07072008000400019 
Guimarães, A.G.C., \& Anjos, A. C. Y. (2012). Caracterização sociodemográfica e avaliação da qualidade de vida em mulheres com câncer de mama em tratamento quimioterápico adjuvante. Revista Brasileira de Cancerologia, 58(4), 581-592.

Huang, H.P., Chen, M.L., Liang, J., \& Miaskowski, C. (2014). Changes in and predictors of severity of fatigue in women with breast cancer: a longitudinal study. International $\begin{array}{llll}\text { Journal of Nursing } & \text { Studies, } & \text { 51(4), }\end{array}$ https://doi.org/10.1016/j.ijnurstu.2013.09.003

Husebo, A.M.L., Dyrstad, S.M., Mjaaland, I., Soreide, J.A., \& Bru, E. (2014) Effects of scheduled exercise on cancer-related fatigue in women with early breast cancer. Scientific World Journal, 1-9. https://doi.org/10.1155/2014/271828

Ingram, C., Wessel, J., \& Courneya, K.S. (2010). Women's perceptions of home-based exercise performed during adjuvant chemotherapy for breast cancer. European Journal of Oncology Nursing, 14(3), 238-243. https://doi.org/10.1016/j.ejon.2010.01.027

Jodar, M., Jacquin, J.P., Vallée, J. (2016). Perception of adverse reactions of chemotherapy and hormone therapy by women treated for breast cancer. Therapie, 71(3), 263-273. https://doi.org/10.1016/j.therap.2016.02.025

Junghaenel, D.U., Cohen, J., Schneider, S., Neerukonda, A.R., \& Broderick, J.E. (2015) Identification of distinct fatigue trajectories in patients with breast cancer undergoing adjuvant chemotherapy. Supportive Care in Cancer, 23(9), 2579-2587. https://doi.org/10.1007/s00520-015-2616-x

Levkovich, I., Cohen, M., Pollack, S., Drumea, K., \& Fried, G. (2015) Cancer-related fatigue and depression in breast cancer patients postchemotherapy: Different associations with optimism and stress appraisals. Palliative \& Supportive Care, 13(5), 1141-1151. https://doi.org/10.1017/S147895151400087X

Liu, L., Mills, P.J., Rissling, M., Fiorentino, L., Natarajan, L., Dimsdale, J.E., Sadler, G. R., Parker, B.A., \& Ancoli-Israel, S. (2012). Fatigue and Sleep Quality are Associated with Changes in Inflammatory Markers in Breast Cancer Patients Undergoing Chemotherapy. Brain, Behavior and Immunity, 26(5), 706-713. https://doi.org/10.1016/j.bbi.2012.02.001

Machado, S.M., \& Sawada, N.O. (2008) Avaliação da qualidade de vida de pacientes oncológicos em tratamento quimioterápico adjuvante. Texto \& Contexto - Enfermagem, $17(4), 750-757$.

Manir, K.S., Bhadra, K., Kumar, G., Manna, A., Patra, N.B., \& Sarkar, S.K. (2012). Fatigue in breast cancer patients on adjuvant treatment: course and prevalence. Indian Journal of Palliative Care, 18(2), 109-116.

Mao, J.J., Farrar, J.T., Bruner, D., Zee, J., Bowman, M., Seluzicki, C., DeMichele, A., \& Xie, S.X. (2014). Electroacupuncture for fatigue, sleep, and psychological distress in breast cancer patients with aromatase inhibitor-related arthralgia: A randomized trial. Cancer, 120(23), 3744-3751. https://doi.org/10.1002/cncr.28917 
Martins, L.C., Filho, C.F., Giglio, A.D., Munhoes, D.A., Trevizan, L.L.B., Herbst, L.G., Viera, M. C., Taranto, P., \& Pachon, S. C. (2009). Desempenho profissional ou doméstico das pacientes em quimioterapia para câncer de mama. Revista da Associação Médica Brasileira, 55(2), 158-162. https://doi.org/10.1590/S0104-42302009000200019

Mendes, K.D.S., Silveira, R.C.D.C.P., \& Galvão, C.M. (2008). Revisão integrativa: método de pesquisa para a incorporação de evidências na saúde e na enfermagem. Texto \& Contexto - Enfermagem, 17(4), 758-764. https://doi.org/10.1590/S010407072008000400018

Meneses-Echávez J.F., González-Jiménez, E., Correa-Bautista, J.E., Ramírez-Vélez, R., \& Valle, J.S.R. (2015). Effectiveness of physical exercise on fatigue in cancer patients during active treatment: a systematic review and meta-analysis. Cadernos de Saúde Pública, 31(4), 667-681. https://doi.org/10.1590/0102-311X00114414

National Cancer Institute (2015, May). Fatigue (PDQ®): Patient Version. Retrieved in Jan 22, 2017, from https://www.cancer.gov/about-cancer/treatment/sideeffects/fatigue/fatigue-pdq.

Nicolussi, A.C., \& Sawada N.O. (2011). Qualidade de vida de pacientes com câncer de mama em terapia adjuvante. Revista Gaúcha de Enfermagem, 32(4), 759-766. https://doi.org/10.1590/S1983-14472011000400017

Oliveira, C.M.B., Sakata, R.K., Issy, A.M., Gerola, L.R., \& Salomão, R. (2011). Citocinas e Dor. Revista Brasileira de Anestesiologia, 61(2), 255-265.

Panobianco, M.S., Magalhães, P.A.P.D., Soares, C.R., Sampaio, B.A.L., Almeida, A.M.D., \& Gozzo, T.O. (2012). Prevalência de depressão e fadiga em um grupo de mulheres com câncer de mama. Revista Eletrônica de Enfermagem, 14(3), 532-540. https://doi.org/10.5216/ree.v14i3.14409

Ratcliff, C.G., Lam, C.Y., Arun, B., Valero, V., \& Cohen, L. (2014) Ecological momentary assessment of sleep, symptoms, and mood during chemotherapy for breast cancer. Psycho-Oncology, 23(11), 1220-1228. https://doi.org/10.1002/pon.3525

Silveira, S.C., \& Zago, M.M.F. (2006). Pesquisa brasileira em enfermagem oncológica: uma revisão integrativa. Revista Latino-Americana de Enfermagem,14(4), 614-619. https://doi.org/10.1590/S0104-11692006000400021

Smith, A.K., Conneely, K.N., Pace, T.W.W., Mister, D., Felger, J.C., Kilaru, V., Akel, M.J., Vertino, P.M. Miller, A.H., \& Torres, M.A. (2014). Epigenetic changes associated with inflammation in breast cancer patients treated with chemotherapy. Brain, Behavior and Immunity, 38, 227-236. https://doi.org/10.1016/j.bbi.2014.02.010

Soares, C.B., Hoga, L.A.K., Peduzzi, M., Sangaleti, C., Yonekura, T., \& Silva, D.R.A.D. (2014). Revisão Integrativa: conceitos e métodos utilizados na enfermagem. Revista da Escola de Enfermagem da USP, 48(2), 335-345. https://doi.org/10.1590/S00806234201400002000020 
Taso, C.J., Lin, H.S., Lin, W.L., Chen, S.M., Huang, W.T., \& Chen, S.W. (2014). The effect of yoga exercise on improving depression, anxiety, and fatigue in women with breast cancer: a randomized controlled trial. Journal of Nursing Research, 22(3), 155-164. https://doi.org/10.1097/jnr.0000000000000044

Travier, N., Velthuis, M.J., Bisschop, C.N.S., Bujis, B.V.D., Monninkhof, E.M., Backx, F., Los, M., Erdkamp, F., Bloemendal, H.J., Rodenhuis, C., Roos, M A.J., Verhaar, M., Huinink, D.B., Van Der Wall, E., Peeters, P.H.M., \& May, A. M. (2015). Effects of an 18-week exercise programme started early during breast cancer treatment: a randomised controlled trial. BMC Medicine, 13:121. https://doi.org/10.1186/s12916-015-0362-z

Ursi E.S. (2005). Prevenção de lesões de pele no perioperatório: revisão integrativa da literatura. Tese de doutorado. Ribeirão Preto: Escola de Enfermagem de Ribeirão Preto, Universidade de São Paulo, Ribeirão Preto, SP, Brasil.

Van Vulpen, J.K., Peeters, P.H.M., Velthuis, M.J., Wall, E.V.D., \& May, A.M. (2016). Effects of physical exercise during adjuvant breast cancer treatment on physical and psychosocial dimensions of cancer-related fatigue: A meta-analysis. Maturitas, 85, 104111. https://doi.org/10.1016/j.maturitas.2015.12.007

Vincent, F., Labourey, J.L., Leobon, S., Antonini, M.T., Lavau-Denes, S., \& TubianaMathieu, N. (2013). Effects of a home-based walking training program on cardiorespiratory fitness in breast cancer patients receiving adjuvant chemotherapy: a pilot study. European Journal of Physical and Rehabilitation Medicine, 49(3), 319-329.

Wang, G., Wang, S., Jiang, P., \& Zeng, C. Effect of yoga on cancer related fatigue in breast cancer patients with chemotherapy. Journal of Central South University (Medical Sciences), 39(10), 1077-1082.

Whittemore, R., \& Knafl, K. (2005). The integrative review: updated methodology. Journal of Advanced Nursing, 52(5), 546-553. https://doi.org/10.1111/j.1365-2648.2005.03621.x

\section{Os autores:}

Anna Cláudia Yokoyama dos Anjos, doutora em Ciências pela Escola de Enfermagem de Ribeirão Preto da Universidade de São Paulo, professora adjunto IV do curso de Graduação em Enfermagem da Faculdade de Medicina da Universidade Federal de Uberlândia. E-mail: annaclaudia1971@ gmail.com.

Cristiane Soares Campos possui graduação em Enfermagem pela Universidade Federal de Uberlândia. Mestranda em Ciências da Saúde pela Universidade Federal de Uberlândia. E-mail: cristianecampos08@gmail.com

Nayara Ferreira Cunha possui graduação em Enfermagem pela Universidade Federal de Uberlândia e Mestrado em Ciências da Saúde pela Universidade Federal de Uberlândia. E-mail: nayara.nfc@ gmail.com

Carine Ferreira Lopes graduanda em Enfermagem pela Universidade Federal de Uberlândia. E-mail: carinelopesenf@gmail.com 
Luana Luiza Alves graduanda em Enfermagem pela Universidade Federal de Uberlândia. E-mail: luanaluiza94@gmail.com.

Juliana Pena Porto, doutora em Microbiologia pela Universidade Federal de Uberlândia, professora adjunto III do curso de Graduação em Enfermagem da Faculdade de Medicina da Universidade Federal de Uberlândia. E-mail: ju-nana@hotmail.com.

\section{Endereço para correspondência:}

Prof ${ }^{a}$ Dr ${ }^{a}$ Anna Cláudia Yokoyama dos Anjos

Universidade Federal de Uberlândia

Curso de Graduação em Enfermagem da Faculdade de Medicina

Avenida Pará, 1720, Campus Umuarama, Bloco 2U, Sala 21.

CEP: 38400-902 - Bairro Umuarama. Uberlândia, MG, Brasil.

E-mail: annaclaudia1971@ gmail.com.

Recebido em: 17/10/2017

Aprovado em: 27/12/2017 